\title{
Chiral Sum Rules to Second Order in Quark Mass
}

\author{
Eugene Golowich \\ Department of Physics and Astronomy, University of Massachusetts \\ Amherst MA 01003 USA \\ Joachim Kambor \\ Institut für Theoretische Physik, Universität Zürich \\ CH-8057 Zürich, Switzerland
}

\begin{abstract}
A new calculation of the isospin and hypercharge axialvector current propagators $\left(\Delta_{\mathrm{A} 33}^{\mu \nu}\left(q^{2}\right)\right.$ and $\left.\Delta_{\mathrm{A} 88}^{\mu \nu}\left(q^{2}\right)\right)$ to two loops in $S U(3) \times S U(3)$ chiral perturbation theory is used to derive chiral spectral function sum rules valid to second order in the light quark masses. Explicit forms are given for the threepion isospin axialvector spectral functions at low energy and application of the sum rules to the determination of counterterms of the chiral lagrangian is discussed.
\end{abstract}

Chiral sum rules were introduced some thirty years ago by Weinberg, who used chiral symmetry to derive two sum rules valid in the limit of massless light quarks. [1] The derivation of several other sum rules soon followed, likewise valid only in the chiral limit. 22.3] These efforts predated the rise of quantum chromodynamics (QCD) as a theory of the strong interactions. A subsequent QCD derivation of the Weinberg sum rules revealed that the first could be extended to the real world of nonzero $u, d, s$ quark mass, but not the second. [4] Due to the analytic intractability of low energy QCD, theoretical understanding of the chiral sum rules away from the chiral limit has been limited. In this Letter we show how chiral perturbation theory (ChPT) [0, 6] can be used to obtain a number of chiral sum rules, mostly new, to second order in quark mass.

The derivation is made possible by a recent calculation of axialvector current propagators through two-loop order in ChPT. [7] This leads to both the derivation of spectral function sum rules for nonzero quark mass and the prediction of axialvector spectral functions at low energy. It also suggests an application of the sum rules to evaluation of $\mathcal{O}\left(p^{6}\right)$ counterterms which appear as part of the ChPT procedure. [8]

The $S U(3)$ axialvector current propagators are defined as

$$
\Delta_{\mathrm{A} a b}^{\mu \nu}\left(q^{2}\right) \equiv i \int d^{4} x e^{i q \cdot x}\left\langle 0\left|T\left(A_{a}^{\mu}(x) A_{b}^{\nu}(0)\right)\right| 0\right\rangle \quad(a, b=1, \ldots, 8)
$$

and have spectral content

$$
\frac{1}{\pi} \mathcal{I} m \Delta_{\mathrm{A} a b}^{\mu \nu}\left(q^{2}\right)=\left(q^{\mu} q^{\nu}-q^{2} g^{\mu \nu}\right) \rho_{\mathrm{A} a b}^{(1)}\left(q^{2}\right)+q^{\mu} q^{\nu} \rho_{\mathrm{A} a b}^{(0)}\left(q^{2}\right)
$$


where $\rho_{\mathrm{A} a b}^{(1)}$ and $\rho_{\mathrm{A} a b}^{(0)}$ are the spin-one and spin-zero spectral functions. The tensor structure of Eq. (2) motivates the decomposition usually adopted in the literature,

$$
\Delta_{\mathrm{A} a b}^{\mu \nu}\left(q^{2}\right)=\left(q^{\mu} q^{\nu}-q^{2} g^{\mu \nu}\right) \Pi_{\mathrm{A} a b}^{(1)}\left(q^{2}\right)+q^{\mu} q^{\nu} \Pi_{\mathrm{A} a b}^{(0)}\left(q^{2}\right)
$$

where $\Pi_{\mathrm{A} a b}^{(1)}$ and $\Pi_{\mathrm{A} a b}^{(0)}$ are respectively the spin-one and spin-zero axialvector polarization functions. As regards flavor notation, we consider $a=b=3$ (isospin), $a=b=8$ (hypercharge) and understand throughout that $a a=33,88$ is not summed. Determination of the axialvector current propagators in ChPT [9] proceeds by introducing axialvector sources into the chiral lagrangians and calculating all amplitudes which connect single source external states. The procedure is basically as described in Ref. [10.

We summarize briefly the results through two-loop order, suppressing flavor labelling for simplicity. For the tree-level propagator, one finds

$$
\Delta_{\mathrm{A}, \mu \nu}^{(\mathrm{tree})}\left(q^{2}\right)=F_{0}^{2} g_{\mu \nu}-\frac{F_{0}^{2}}{q^{2}-m^{2}} q_{\mu} q_{\nu}
$$

where $m$ and $F_{0}$ are the mass and decay constant parameters to leading order in the chiral expansion. The proper behavior is seen to occur in the chiral limit $m \rightarrow 0$, where the propagator takes on a purely spin-one ('transverse') form consistent with current conservation $\partial_{\mu} A^{\mu}=0$. Rewriting the above in terms of the tensor structure of Eq. (2) yields

$$
\Delta_{\mathrm{A}, \mu \nu}^{(\text {tree })}\left(q^{2}\right)=-F_{0}^{2}\left[\frac{1}{q^{2}}\left(q_{\mu} q_{\nu}-q^{2} g_{\mu \nu}\right)+\frac{m^{2}}{q^{2}\left(q^{2}-m^{2}\right)} q_{\mu} q_{\nu}\right] .
$$

There are kinematic poles at $q^{2}=0$ in both the spin-one and spin-zero polarization functions, although the sum $\Pi_{\mathrm{A}}^{(1)}+\Pi_{\mathrm{A}}^{(0)}$ is free of such poles.

We have determined the propagator through two-loop order (i.e. summing over tree, one-loop and two-loop contributions) to have the structure

$$
\Delta_{\mathrm{A}, \mu \nu}\left(q^{2}\right)=\left(F^{2}+\hat{\Pi}_{\mathrm{A}}^{(0)}\left(q^{2}\right)\right) g_{\mu \nu}-\frac{F^{2}}{q^{2}-M^{2}} q_{\mu} q_{\nu}+\left(2 L_{10}^{\mathrm{r}}-4 H_{1}^{\mathrm{r}}+\hat{\Pi}_{\mathrm{A}}^{(1)}\left(q^{2}\right)\right)\left(q_{\mu} q_{\nu}-q^{2} g_{\mu \nu}\right)
$$

where $F^{2}, M^{2}$ are now renormalized at two-loop level, $L_{10}^{\mathrm{r}}, H_{1}^{\mathrm{r}}$ are $\mathcal{O}\left(p^{4}\right)$ counterterms which appear in the one-loop analysis and $\hat{\Pi}_{\mathrm{A}}^{(0,1)}\left(q^{2}\right)$ are finite two-loop functions. The process of deriving Eq. (6), describing the renormalization procedure and displaying the various formulae is quite lengthy and will be deferred to another setting. [7] However, $\hat{\Pi}_{\mathrm{A}}^{(0)}\left(q^{2}\right)$ can be shown to vanish in the limit of zero quark mass and thus the above expression has the proper chiral behavior. The relation between the amplitudes appearing in Eq. (3) with those in Eq. (6) is

\footnotetext{
${ }^{1}$ The quantity $H_{1}^{\mathrm{r}}$ is regularization dependent, as are analogous terms in $\hat{\Pi}^{(0)}(0)$. However, such unphysical contributions are absent from the spectral function sum rules.
} 


$$
\begin{aligned}
& \Pi_{\mathrm{A}}^{(1)}\left(q^{2}\right)=2 L_{10}^{\mathrm{r}}-4 H_{1}^{\mathrm{r}}+\hat{\Pi}_{\mathrm{A}}^{(1)}\left(q^{2}\right)-\frac{F^{2}+\hat{\Pi}_{\mathrm{A}}^{(0)}\left(q^{2}\right)}{q^{2}} \\
& \Pi_{\mathrm{A}}^{(0)}\left(q^{2}\right)=\frac{\hat{\Pi}_{\mathrm{A}}^{(0)}\left(q^{2}\right)}{q^{2}}-\frac{F^{2} M^{2}}{q^{2}\left(q^{2}-M^{2}\right)}
\end{aligned}
$$

We now turn to some implications of the two-loop analysis.

In chiral perturbation theory, the axialvector spectral functions $\rho_{\mathrm{A} a b}^{(0,1)}$ first receive contributions at chiral order $p^{6}$. Their threshold behavior can be deduced directly from Eq. (2) via the imaginary parts of the two-loop propagators. Such contributions arise entirely from the set of ('sunset') diagrams having three-particle intermediate states. Figures 1,2 display the predicted low energy behavior of the three-pion contributions to the isospin spectral functions $\rho_{\mathrm{A} 33}^{(1)}[3 \pi]$ and $\rho_{\mathrm{A} 33}^{(0)}[3 \pi]$. The spectral function $\rho_{\mathrm{A} 33}^{(1)}[3 \pi]$ has been inferred phenomenologically from $3 \pi$ emission in tau decay over much of the allowed energy range, [1] although more data near threshold is required to decisively test the ChPT prediction. To our knowledge no phenomenological determination of $\rho_{\mathrm{A} 33}^{(0)}[3 \pi]$ yet exists, and we will address this topic in a future work.

An independent procedure for calculating the spectral functions is to employ unitarity by inserting all possible three-pion intermediate states in the two-point functions. [12], [13] We find the two-loop and unitarity schemes to yield precisely the same results. This serves as a highly nontrivial check on the correctness of the two-loop functions $\hat{\Pi}_{\mathrm{A}}^{(0,1)}\left(q^{2}\right)$ as well as on the constituitive relations in Eq. (1).

The derivation of chiral sum rules proceeds by first obtaining dispersion theoretic expressions for the various polarization functions. The asymptotic behavior $(s \rightarrow \infty)$ of the vector and axialvector spectral functions which follows from QCD is given by [四, [14]

$$
\begin{aligned}
& \rho_{\text {Vaa }}^{(1)}(s) \sim \mathcal{O}(1), \quad \rho_{\text {A } a a}^{(1)}(s) \sim \mathcal{O}(1), \quad \rho_{\text {A } a a}^{(0)}(s) \sim \mathcal{O}\left(s^{-1}\right), \quad\left(\rho_{\mathrm{A} 33}^{(1)}-\rho_{\mathrm{A} 88}^{(1)}\right)(s) \sim \mathcal{O}\left(s^{-1}\right) \\
& \left(\rho_{\text {Vaa }}^{(1)}-\rho_{\text {A } a a}^{(1)}\right)(s) \sim \mathcal{O}\left(s^{-1}\right), \quad\left(\rho_{\text {Vaa }}^{(1)}-\rho_{\text {A } a a}^{(1)}-\rho_{\text {A } a a}^{(0)}\right)(s) \sim \mathcal{O}\left(s^{-2}\right)
\end{aligned}
$$

where $\rho_{\text {Vaa }}^{(1)}$ is defined analogously to $\rho_{\text {Aaa }}^{(1)}(c f$ Eq. (2) $)$ and $\rho_{\text {Vaa }}^{(0)}$ vanishes since we assume isospin symmetry. The information in Eq. (8) can be used, together with analyticity and the corresponding asymptotic behavior of the polarization functions, to derive dispersion relations for the vector and axialvector polarization functions. The dispersion relations occur in three classes, beginning with those which combine vector and axialvector amplitudes, e.g.

$$
\left(\Pi_{\mathrm{V} a a}^{(1)}-\Pi_{\mathrm{A} a a}^{(1)}-\Pi_{\mathrm{A} a a}^{(0)}\right)\left(q^{2}\right)=\int_{0}^{\infty} d s \frac{\left(\rho_{\mathrm{V} a a}^{(1)}-\rho_{\mathrm{A} a a}^{(1)}-\rho_{\mathrm{A} a a}^{(0)}\right)(s)}{s-q^{2}-i \epsilon} .
$$

Due to the highly convergent behavior of $\left(\rho_{\text {Vaa }}^{(1)}-\rho_{\text {Aaa }}^{(1)}-\rho_{\text {Aaa }}^{(0)}\right)(s)$ at large $s$, this combination of polarization functions is 'superconvergent', i.e. the first moment $q^{2}\left(\Pi_{\text {Vaa }}^{(1)}-\Pi_{\text {Aaa }}^{(1)}-\Pi_{\text {Aaa }}^{(0)}\right)\left(q^{2}\right)$ also obeys an unsubtracted dispersion relation. Then there are dispersion relations for the axialvector polarization functions of a given flavor, e.g.

$$
q^{2} \Pi_{\mathrm{A} a a}^{(0)}\left(q^{2}\right)-\lim _{q^{2}=0}\left(q^{2} \Pi_{\mathrm{A} a a}^{(0)}\left(q^{2}\right)\right)=q^{2} \int_{0}^{\infty} d s \frac{\rho_{\mathrm{A} a a}^{(0)}(s)}{s-q^{2}-i \epsilon}
$$


where we work with $q^{2} \Pi_{\mathrm{Aaa}}^{(0)}\left(q^{2}\right)$ due to the presence of $q^{2}=0$ kinematic poles. The quantity $q^{2} \Pi_{\text {Aaa }}^{(1)}\left(q^{2}\right)$ obeys an analogous relation. Finally, there are dispersion relations for $S U(3)$ breaking combinations, such as $\left(\Pi_{\mathrm{A} 33}^{(1)}+\Pi_{\mathrm{A} 33}^{(0)}-\Pi_{\mathrm{A} 88}^{(1)}-\Pi_{\mathrm{A} 88}^{(0)}\right)\left(q^{2}\right)$ and $q^{2}\left(\Pi_{\mathrm{A} 33}^{(1)}-\Pi_{\mathrm{A} 88}^{(1)}\right)\left(q^{2}\right)$.

Sum rules are obtained by evaluating arbitrary derivatives of such relations at $q^{2}=0$. We consider first the two most well-known examples. Thus, evaluation at $q^{2}=0$ of the dispersion relation for $q^{2}\left(\Pi_{\text {Vaa }}^{(1)}-\Pi_{\text {Aaa }}^{(1)}-\Pi_{\text {A } a a}^{(0)}\right)\left(q^{2}\right)$ yields Weinberg's first sum rule, [1]

$$
F_{a}^{2}=\int_{0}^{\infty} d s\left(\rho_{\mathrm{V} a a}^{(1)}-\rho_{\mathrm{A} a a}^{(1)}-\bar{\rho}_{\mathrm{A} a a}^{(0)}\right)(s)
$$

where we have defined $\bar{\rho}_{\text {Aaa }}^{(0)}(s) \equiv \rho_{\text {Aaa }}^{(0)}(s)-F_{a}^{2} \delta\left(s-M_{a}^{2}\right)$. Note that the sum rule is now evaluated away from the chiral limit. Likewise Eq. (9) at $q^{2}=0$ yields the inverse-moment sum rule [3,15]

$$
\left(\Pi_{\mathrm{V} a a}^{(1)}-\Pi_{\mathrm{A} a a}^{(1)}-\Pi_{\mathrm{A} a a}^{(0)}\right)(0)=\int_{0}^{\infty} d s \frac{\left(\rho_{\mathrm{V} a a}^{(1)}-\rho_{\mathrm{A} a a}^{(1)}-\rho_{\mathrm{A} a a}^{(0)}\right)(s)}{s},
$$

which now includes two-loop contributions on the left-hand-side (LHS).

More generally, entire sequences of sum rules are derivable, such as those for a given flavor,

$$
\begin{aligned}
& \frac{1}{n !}\left[\frac{d}{d q^{2}}\right]^{n}\left(\Pi_{\mathrm{V} a a}^{(1)}-\Pi_{\mathrm{A} a a}^{(1)}-\Pi_{\mathrm{A} a a}^{(0)}\right)(0)=\int_{0}^{\infty} d s \frac{\left(\rho_{\mathrm{V} a a}^{(1)}-\rho_{\mathrm{A} a a}^{(1)}-\rho_{\mathrm{A} a a}^{(0)}\right)(s)}{s^{n+1}} \quad(n \geq 0), \\
& \frac{1}{n !}\left[\frac{d}{d q^{2}}\right]^{n} \hat{\Pi}_{\mathrm{A} a a}^{(0)}(0)=\int_{0}^{\infty} d s \frac{\bar{\rho}_{\mathrm{A} a a}^{(0)}(s)}{s^{n}} \quad(n \geq 1), \\
& \frac{1}{(n-1) !}\left[\frac{d}{d q^{2}}\right]^{n-1} \hat{\Pi}_{\mathrm{A} a a}^{(1)}(0)-\frac{1}{n !}\left[\frac{d}{d q^{2}}\right]^{n} \hat{\Pi}_{\mathrm{A} a a}^{(0)}(0)=\int_{0}^{\infty} d s \frac{\rho_{\mathrm{A} a a}^{(1)}(s)}{s^{n}} \quad(n \geq 2) .
\end{aligned}
$$

A sequence of sum rules explicitly involving broken $S U(3)$ is

$$
\frac{1}{n !}\left[\frac{d}{d q^{2}}\right]^{n}\left(\Pi_{\mathrm{A} 33}^{(1)}+\Pi_{\mathrm{A} 33}^{(0)}-\Pi_{\mathrm{A} 88}^{(1)}-\Pi_{\mathrm{A} 88}^{(0)}\right)(0)=\int_{0}^{\infty} d s \frac{\left(\rho_{\mathrm{A} 33}^{(1)}+\rho_{\mathrm{A} 33}^{(0)}-\rho_{\mathrm{A} 88}^{(1)}-\rho_{\mathrm{A} 88}^{(0)}\right)(s)}{s^{n+1}}
$$

with $n \geq 0$.

Various applications of chiral sum rules have appeared in the literature since their introduction. These range from obtaining relations between the spectra of vector and axialvector mesons [1] to applying the full battery of data and theory inputs to phenomenologically test the Weinberg sum rules in their zero mass setting. [16] Here we show how to obtain numerical estimates for certain finite $\mathcal{O}\left(p^{6}\right)$ counterterms associated with the ChPT renormalization procedure. 17 Recall that in a two-loop calculation the leading divergences must be cancelled by $\mathcal{O}\left(p^{6}\right)$ counterterms $\left\{B_{k}\right\}$. Each such counterterm can be expressed in dimensional regularization as

$$
B_{k}=\mu^{2(d-4)} \sum_{n=2}^{-\infty} B_{k}^{(n)}(\mu) \bar{\lambda}^{n}=\mu^{2(d-4)}\left[B_{k}^{(2)}(\mu) \bar{\lambda}^{2}+B_{k}^{(1)}(\mu) \bar{\lambda}+B_{k}^{(0)}(\mu)+\ldots\right]
$$


where $\bar{\lambda}$ is the singular quantity

$$
\bar{\lambda} \equiv \frac{1}{16 \pi^{2}}\left[\frac{1}{d-4}-\frac{1}{2}(\log 4 \pi-\gamma+1)\right] .
$$

The $\left\{B_{k}^{(2)}(\mu)\right\}$ and $\left\{B_{k}^{(1)}(\mu)\right\}$ are chosen to subtract off all divergences, and a large number of these so-called $\beta$-functions is thereby determined. [7] However, the physical result will contain the finite quantities $\left\{B_{k}^{(0)}(\mu)\right\}$. 2 Like the renormalized electron charge and mass in QED, these finite counterterms must somehow be determined from experiment. Thus, for example consider the sum rule of Eq. (15) with $n=2$ and isospin flavor,

$$
4\left(2 B_{32}^{(0)}-B_{33}^{(0)}\right)(\mu)-\frac{1}{3072 \pi^{4} F_{\pi}^{2}}\left(0.204+\log \frac{M_{\pi}^{2}}{\mu^{2}}+\frac{5}{4} \log \frac{M_{K}^{2}}{\mu^{2}}\right)=\int_{0}^{\infty} d s \frac{\rho_{\mathrm{A} 33}^{(1)}(s)}{s^{2}},
$$

where the first term inside the parentheses on the LHS arises from a scale-independent two-loop contribution. From the renormalization procedure, one knows already that $\left(2 B_{32}^{(2)}-B_{33}^{(2)}\right)(\mu)=0$ and $\left(2 B_{32}^{(1)}-B_{33}^{(1)}\right)(\mu)=3 / 1024 \pi^{2} F_{\pi}^{2}$. To estimate $\left(2 B_{32}^{(0)}-B_{33}^{(0)}\right)(\mu)$, we approximate the spectral function in terms of the $a_{1}$ resonance contribution taken in narrow width approximation, $\rho_{\mathrm{A} 33}^{(1)}(s) \simeq g_{a_{1}} \delta\left(s-M_{a_{1}}^{2}\right)$. From the fit of Ref. [16] and adopting the renormalization scale $\mu=M_{a_{1}}$, we obtain $\left(2 B_{32}^{(0)}-B_{33}^{(0)}\right)\left(M_{a_{1}}\right) \simeq$ $0.0030 \mathrm{GeV}^{-2}$. The $\mathcal{O}\left(p^{6}\right)$ counterterm dominates the other terms on the LHS of Eq. (19), showing the importance of a full ChPT calculation as compared to a chiral-log treatment. A more thorough phenomenological analysis will involve use of the entire spectrum and include error bars in the final estimate. However, this simple example serves to demonstrate the general procedure.

To conclude, a new two-loop ChPT calculation of axialvector current propagators, as embodied by Eq. (6), has been performed. In addition to yielding a complete two-loop renormalization of the pion and eta masses and decay constants, it has led to predictions for axialvector spectral functions and to the derivation of spectral function sum rules. An application of the sum rules to determine finite $\mathcal{O}\left(p^{6}\right)$ counterterms has been provided. Additional work will involve careful analysis of the existing database to provide as precise a determination of the counterterms as experimental uncertainties allow as well as addressing the phenomenological extraction of spectral functions like $\rho_{\mathrm{A} 33}^{(0)}[3 \pi]$.

We thank John Donoghue, Jürg Gasser and Marc Knecht for useful comments. This work was supported by the US National Science Foundation and by Schweizerischer Nationalfonds.

\footnotetext{
${ }^{2}$ The dependence on renormalization scale $\mu$ is known explicitly from renormalization group equations.
} 


\section{REFERENCES}

[1] S. Weinberg, Phys. Rev. Lett. 18 (1967) 507.

[2] T. Das, G.S. Guralnik, V.S. Mathur, F.E. Low and J.E. Young, Phys. Rev. Lett. 18 (1967) 759 .

[3] T. Das, V.S. Mathur and S. Okubo, Phys. Rev. Lett. 19 (1967) 859.

[4] E.G. Floratos, S. Narison and E. de Rafael, Nucl. Phys. B155 (1979) 115.

[5] S. Weinberg, Physica A96, (1979) 327.

[6] J. Gasser and H. Leutwyler, Ann. Phys. 158 (1984) 142.

[7] E. Golowich and J. Kambor, in preparation.

[8] We employ the set of $\mathcal{O}\left(p^{6}\right)$ counterterms prepared by H.W. Fearing and S. Scherer, Phys. Rev. D53 (1996) 315.

[9] For clarity's sake, we emphasize that our analysis is in the standard ChPT and not the generalized ChPT of J. Stern et al, Phys. Rev. D47 (1993) 3814.

[10] E. Golowich and J. Kambor, Nucl. Phys. B447, (1995) 373.

[11] For example, see A. Höcker, Vector and Axila-Vector Spectral Functions and QCD, Univ. de Paris-Sud preprint (March 1997), hep-ex/9703004; R. Balest et al (CLEO collaboration), Phys. Rev. Lett. 75 (1995) 3809.

[12] This approach already appears for the $\mathcal{O}\left(p^{6}\right)$ isospin vector current spectral function in Appendix B of Ref. [10].

[13] An $\mathcal{O}\left(p^{8}\right)$ determination of the isospin axialvector current spectral function is given by G. Colangelo, M. Finkemeier and R. Urech, Phys. Rev. D54 (1996) 4403.

[14] E. Braaten, S. Narison and A. Pich, Nucl. Phys. B373 (1992) 581; M. Jamin and M. Münz, Z. Phys. C60 (1993) 569.

[15] See also J. Gasser and H. Leutwyler, Nucl. Phys. B250 (1985) 465; G. Ecker, J. Gasser, A. Pich and E. de Rafael, ibid B321 (1989) 311.

[16] J.F. Donoghue and E. Golowich, Phys. Rev. D49 (1994) 1513.

[17] An analogous procedure has been explored for the vector propagator case by E. Golowich and J. Kambor, Phys. Rev. D53 (1996) 2651. 


\section{FIGURE CAPTIONS}

1. Three-pion contribution to spin-one isospin spectral function.

2. Three-pion contribution to spin-zero isospin spectral function. 


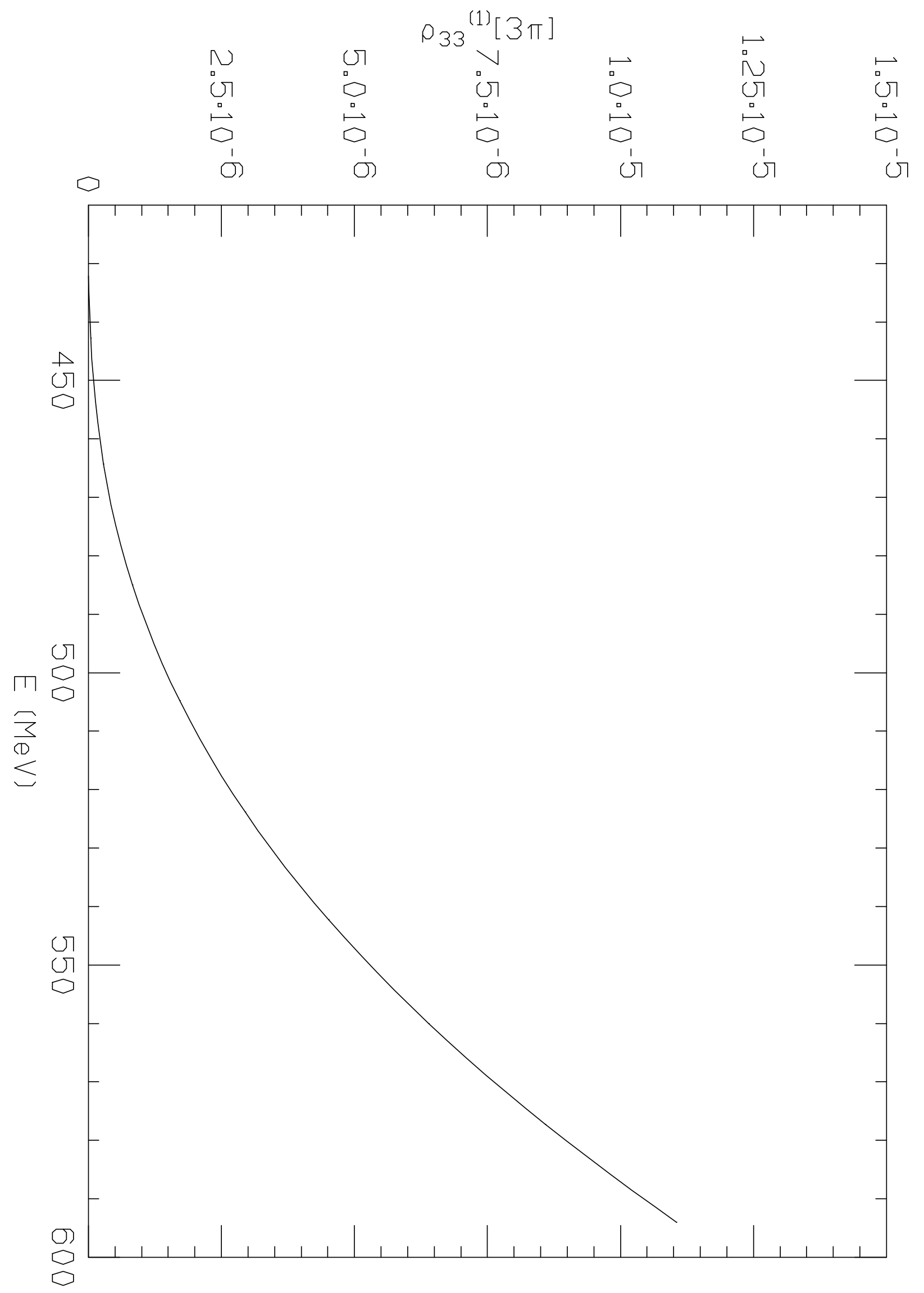




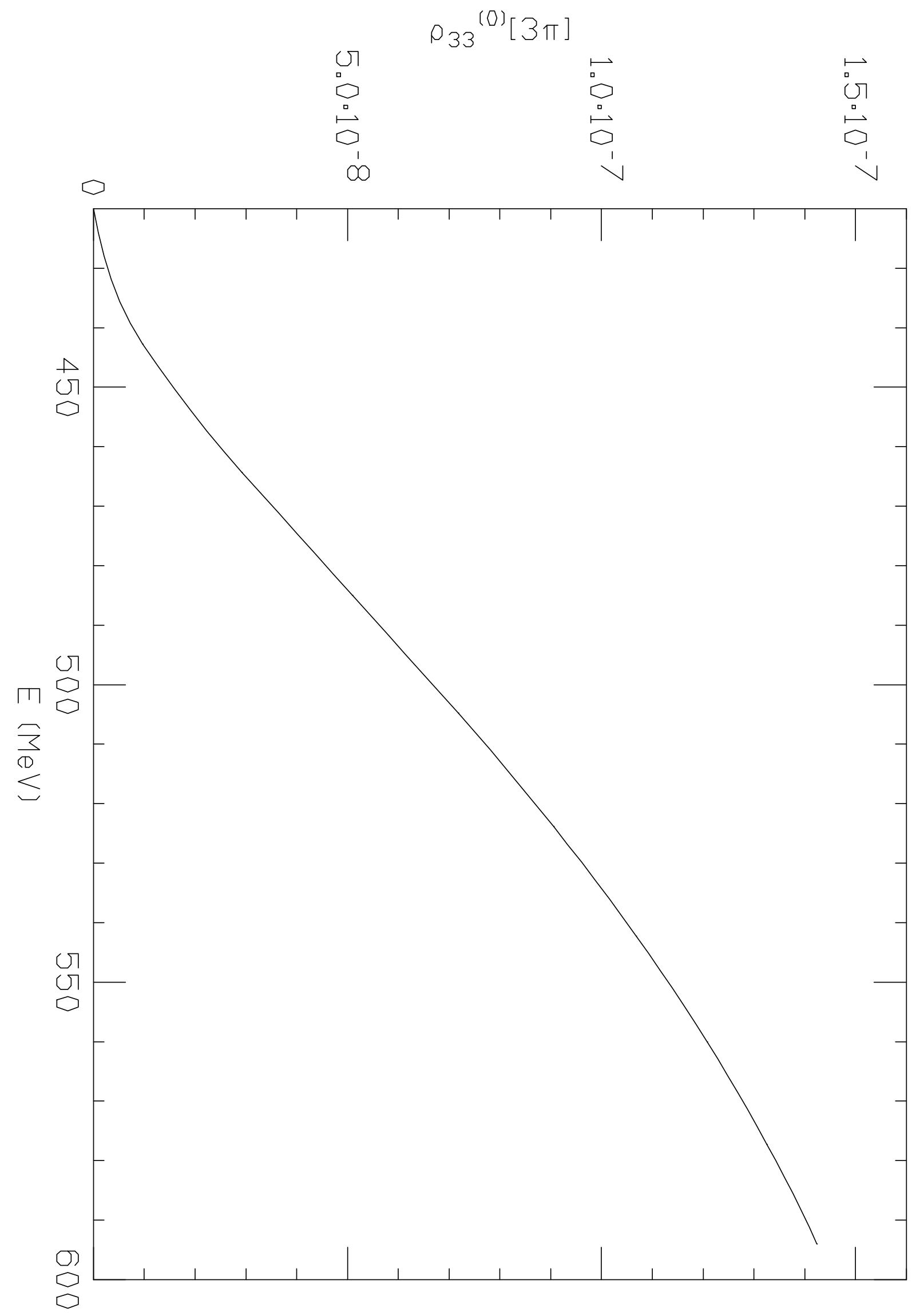

